\section{Euro coins and the}

\section{potential risk of nickel}

\section{allergy}

Paul-Guy Fournier', Thomas R. Govers', and Anne Brun ${ }^{3}$

${ }^{1}$ Laboratoire de Spectroscopie de Translation, Université Paris-

Sud, Orsay

${ }^{2}$ Aecono Consulting, Paris

${ }^{3}$ Service Médical du Travail, Université Paris-Sud, Orsay

\section{The concern about nickel in coins}

$T^{\text {HE }}$ choice of design and material to be used in the manufac$I$ ture of the European common coinage has been the subject of much debate [1]. The incorporation of nickel, in particular, was questioned because of its possible contribution to contact dermatitis: nickel allergy is reported to affect more than $10 \%$ of women and several \% of men in industrialised countries [2]. A compromise was reached by limiting nickel to the two highest denominations: the 1 - and $2 €$ coins. The surface of both combines a white copper-nickel alloy nickel allergy is (Cu75Ni25) with a yellow nickelhaving a yellow outer ring and reported to affect white centre, the $2 €$ having a white more than $10 \%$ of outer ring and a yellow centre. The six other euro coins do not contain nickel.

women and Several recent articles dealing with leaching experiments or patch tests have renewed attention to the potential allergy risk of the 1 - and $2 €$ pieces [2-4]. The work published by Nestle et al. in the September $12^{\text {th }}, 2002$, issue of Nature [4], in particular, has been given widespread public coverage, claiming, for instance, that the "euros break EU allergy directive" [5] and that "anybody who is sensitive to nickel may also wish to handle the coins with care" [6]. At time of writing, an internet query with the keywords "euro + nickel + allergy" brought up close to 900 web pages using a popular search engine. Virtually all of these claim that the euro coins represent a danger in regard to nickel allergy, ignoring the fact that the only two nickel-containing euro denominations release less nickel upon manipulation than pure-nickel or nickel-alloy coins of comparable size [7].

The 1994 EU "nickel directive" [8] concerns objects designed to come into direct and prolonged contact with the skin. The corresponding normalised test procedure, EN1811 [9], measures the amount of nickel dissolved when the object concerned is immersed in synthetic sweat during one week. When one applies this procedure to the $1-$ and $2 €$ coins one detects nickel in amounts comparable to those obtained with other copper-nickel coins and higher than those measured for pure-nickel pieces $[2,4$, $10,11]$. These amounts do indeed exceed the $0.5 \mu \mathrm{g} / \mathrm{cm}^{2} /$ week limit of the "nickel directive". But the directive does not apply to coins, and rightly so: interpolating the release rates obtained, taking into account that the manipulation of a coin typically takes less than three seconds rather than a week, one finds numbers that are several orders of magnitude lower than what is actually observed in tests that simulate the daily manipulation of coins $[11,7]$. The EN1811 test is relevant to long-term solvation upon contact with the skin, but because it does not account for the friction that characterises the manipulation of coins, it is not representative of the amount of nickel transferred to the fingers by the handling of coinage. As anyone can verify when taking a shower, friction is much more efficient in transferring contamination than frictionless solvation.

\section{Manipulation tests}

To take friction into account, we have evaluated metal contamination from coins by means of a simple test that simulates daily handling [7]: participating volunteers count a set of coins by transferring them from one polyethylene container to another, and metal contamination sampled by wiping the fingers with cellulose cloths is analysed by inductively-coupled plasma emission spectroscopy (ICP-OES). Details of the procedure can be found in references [7] and [12].

Table 1 summarizes the results of such tests carried out by 3 people counting three sets of 25 used coins each: bi-metallic euros (12 pieces of $1 €$ and 13 of $2 €$ ), Cu75Ni25 copper-nickel coins (2SFr), and pure-nickel $2 \mathrm{FF}$ pieces. The first two sets were taken from circulation in a non-industrial environment early 2003, and the last in the beginning of 2002. These $2 \mathrm{FF}$ coins had since been stored in a closed polyethylene container. All coins were counted as collected.

\begin{tabular}{|c|c|c|c|c|}
\hline & Ambient $[7]$ & $1 \& 2 €$ & $\mathrm{Cu} 75 \mathrm{Ni} 25$ & $\mathrm{Ni} 100$ \\
\hline $\mathrm{Ni}$ & $3.1 \pm 0.8$ & $0.31 \pm 0.08$ & $0.50 \pm 0.16$ & $0.55 \pm 0.23$ \\
$\mathrm{Cu}$ & $11.4 \pm 3.5$ & $1.95 \pm 0.54$ & $1.77 \pm 0.54$ & $0.16 \pm 0.09$ \\
$\mathrm{Zn}$ & $18.8 \pm 5.8$ & $0.29 \pm 0.09$ & $0.04 \pm 0.03$ & $0.09 \pm 0.06$ \\
\hline \multicolumn{2}{|c|}{ Area 2 faces $\left(\mathrm{cm}^{2}\right)$} & $8.5 \& 10.4$ & 11.8 & 11.0 \\
\hline
\end{tabular}

Aable 1: Average contamination levels resulting from the manipulation of a single used coin. The second data column lists numbers obtained by counting a set of $121 €$ and $132 €$ pieces; the third column was obtained with $252 \mathrm{SFr}$ coins, and the last with $252 \mathrm{FF}$ pieces. All data are expressed in $\mu \mathrm{g}$. Uncertainties are $90 \%$ confidence limits. The first column recalls the average contamination levels found on three fingers as a result of daily activities [7].

Clearly, handling the two nickel-containing euros does transfer significant amounts of nickel to the fingers, but the amounts involved are about $40 \%$ lower than those resulting from the manipulation of pure-nickel or copper-nickel coins of comparable size. The comparison between the euros and the copper-nickel coins agrees well with the compositions averaged over their respective face areas: Cu75Ni15Zn10 for both bi-metallic euros and $\mathrm{Cu} 75 \mathrm{Ni25}$ for the single-alloy coins. Note that a significant amount of copper is transferred when manipulating pure-nickel francs. We ascribe it to the contamination of the pure-nickel pieces upon contact with copper-containing coins in daily usage.

The contamination transferred to the fingers consists mainly of pre-existing species, rather than compounds generated during manipulation [12]. This is illustrated by fig. I which shows the decrease in nickel contamination observed upon repeated manipulation of the same used copper-nickel coin (a Swiss 2SFr) or the same used $2 €$. Handling of a used coin is seen to quite effectively 
remove pre-existing contamination by transferring it to the fingers. This transfer becomes less rapid as the number of manipulations increases, because the remaining nickel species are those which are strongest bound to the coin's surface.

The amount of pre-existing metallic species accessible to contaminate the fingers can be evaluated by rubbing the coins to a shiny polish and determining the amount of metal recovered on the "wipes" used to do so [12]. Such polishing reduces contamination upon subsequent handling by more than a factor 10 , indicating that it removes more than $90 \%$ of accessible pre-existing contamination [12]. The results obtained for several types of nickel containing coins are summarized in table 2 .

\begin{tabular}{|c|c|c|c|}
\hline & $2 €$ & Cu75Ni25 & Ni100 \\
\hline $\mathrm{Ni}$ & $0.48 \pm 0.07$ & $0.97 \pm 0.37$ & $1.1 \pm 0.1$ \\
$\mathrm{Cu}$ & $3.7 \pm 0.5$ & $4.0 \pm 1.1$ & $1.2 \pm 0.7$ \\
$\mathrm{Zn}$ & $0.8 \pm 0.2$ & $0.4 \pm 0.2$ & $0.2 \pm 0.2$ \\
\hline
\end{tabular}

4. Table 2: Metal amounts pre-existing on the surface of used coins and accessible to contamination of the fingers by manipulation. The data are expressed in $\mu \mathrm{g}$ per $\mathrm{cm}^{2}$ and were obtained by dividing the amounts collected when polishing the coins by the geometric surface area of their two faces. The central data column is the average of five different used coins made from Cu75Ni25 copper-nickel alloy. The $2 €$ and pure-nickel (2FF) data are the average of 60 different coins in each case. Uncertainties are $90 \%$ confidence limits.

In this table, the accessible contamination for a single coin has been divided by the geometric surface area of its two faces. The $2 €$ and the pure-nickel $2 \mathrm{FF}$ data are averages pertaining to samples of 60 pieces in each case. The middle column represents the averages of measurements on five different coins made of $\mathrm{Cu} 75 \mathrm{Ni} 25$ copper-nickel. This alloy releases essentially the same amount of nickel per unit area as does pure nickel, in spite of its fourfold reduction in nickel content.

When comparing the euro data with the other copper-nickel coins, the accessible amounts of nickel and copper are found to closely reproduce the surface-averaged alloy compositions. The manipulation tests illustrated by table 1 and the accessibility measurements of table 2 are fully coherent, and neither set of data shows any evidence for enhanced nickel release due to the bimetallic structure of the 1 - and $2 €$ coins, contrary to the strong galvanic effect reported in the case of immersion tests [4,7].

\section{Patch tests}

As nickel allergy results from penetration of the skin by nickel ions $[13,14]$, the question arises what portion of nickel contamination, once transferred to the fingers, will be dissolved before being removed by washing or rubbing hands. We have examined the dissolution rates upon contact with the skin by means of "patch" tests analogous to those employed by Aberer and Kränke [3]. In the present case, we have replaced visual evaluation by quantified measurement of metal release using the same sampling and analysis procedure as in the manipulation tests. To obtain data representative of the coin constituents, the different pieces investigated were previously polished to remove superficial contamination.

Two patch tests were carried out, each with 8 different coins, these being left taped to the skin during 24 and 72 hours, respectively. Subsequently, the tapes and coins were carefully removed, and the imprint on the skin of each individual coin was thoroughly rubbed, each time using fresh cellulose "wipes" which were separately stored in closed polyethylene containers. This sampling procedure was carried out at the University health centre, under medical supervision.

Figure 2 illustrates the imprints observed after $72 \mathrm{~h}$ skin contact of four different coins, identified in the photograph. There are no obvious visual differences between the various pieces, except, perhaps, for the stronger mark left by the copper-nickel Swiss $2 \mathrm{SFr}$ as compared to that due to the $2 €$ coin. The mark left by the nickel-free $0.50 €$ piece (Cu89Al5Zn5Sn1) is visually quite similar to that of the three other coins, made of nickel-alloys, illustrating that much of the observed coloration is due to copper rather than to nickel. There is no resemblance at all to the spectacular visual difference between the Swiss franc and the euro observed upon long-term immersion in artificial sweat $[4,15]$.

In the laboratory, ICP-OES analysis was applied to the "wipes" used to rub the imprints on the arm and also to those employed for sampling metals released on the surfaces of the coins that had been in contact with the skin. The results are summarised in fig.3, where the sum of the metals collected on the skin and on the coins are reported as release rates per $\mathrm{cm}^{2}$ and per week. Expressed in this fashion, the results from the $24 \mathrm{~h}$ experiment, indicated by an asterisk ${ }^{*}$, and from the $72 \mathrm{~h}$ patch test show little difference, indicating that there are no important saturation effects in spite of the limited amount of sweat available to dissolve into.

The data demonstrate a remarkable consistency between coins of similar composition and a close correlation with the surface-averaged proportion of the metals examined. The Swiss, American, and Swedish coins, as well as for the British $10 \mathrm{p}$ are all made of $\mathrm{Cu} 75 \mathrm{Ni} 25$ copper-nickel, and for both constituents the release rates per $\mathrm{cm}^{2}$ too are very similar. The nickel release averages between 20 and $30 \mu$ of nickel per $\mathrm{cm}^{2} /$ week, the area considered being that of one face of the coin. This is about twice as much as the results obtained with the pure-nickel 1FF pieces, in qualitative agreement with prolonged immersion experiments in artificial sweat, which yield an even larger increase in nickel release from copper-nickel as compared to pure nickel $[2,4,10,11]$.

Evolution of nickel contamination averaged over 25 successive manipulations

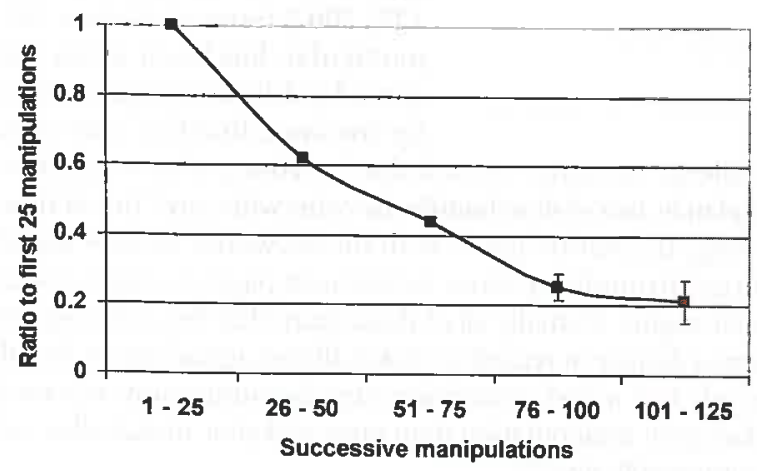

$\Delta$ Fig. 1: Evolution of nickel contamination upon repeated manipulation of the same used coin, whereby the fingers are wiped for metal analysis after each sequence of 25 manipulations. The data are reported as ratios to the quantities obtained for the first sequence, and are the averages of the ratios obtained with used $2 €$ coins and used copper-nickel pieces. The error bars indicate the spread between the two types of coins. 


\section{$0,5 €$ \\ $14 \$$
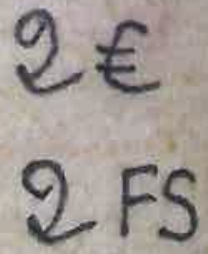

A Fig. 2: Imprints visible just after removing coins that had been taped to the skin during 72 hours.

the copper-nickel and nickel-brass data.

The $0.50 €$ coin, consisting of nickel-free "Nordic Gold", releases copper slightly higher in proportion to its copper content than the copper-nickel coins, indicating a somewhat lower corrosion resistance upon contact with human sweat. Its nickel release rate is small, about $3 \mu \mathrm{g}$ per $\mathrm{cm}^{2}$ and per week, but significantly higher than can be ascribed to nickel impurity in its constituents. This contamination probably results from contact with the nickel-containing 1 - and $2 €$ pieces in daily usage. As the coins were polished at the onset of the present patch tests, the persistence of this metallic cross-contamination reflects relatively strong adhesion to the coin's surface. An analogous explanation probably applies to the copper observed on the pure-nickel French franc and to the zinc on the 50 öre and on the US coins.

\section{Allergic risk}

We have shown above that in experiments representative of the handling of coins, the nickel-containing $1 €$ and $2 €$ coins behave very similarly to single-alloy copper-nickel pieces, in regard both to contamination transfer by friction and to dissolution upon contact with the skin. It is therefore reasonable to assume that the nature of the contamination transferred to the fingers is similar in both cases. When comparing these two euro pieces to other copper-nickel coins, the relative risk of nickel allergy should in that case be in proportion to the amounts of nickel transferred by manipulation. This amount is typically $40 \%$ lower with the 1- and $2 €$ pieces, as reported in table 1 and in ref. [7], suggesting that the two nickel-containing euros represent a lesser risk of nickel allergy than Cu75Ni25 copper-nickel coins of similar size.

The comparison may be less straightforward when comparing the euros with pure-nickel coins, as the fraction of transferred nickel that dissolves before washing or rubbing hands may be different. As illustrated by fig. 3 ,

\section{A more precise the nickel dissolution rate of the} Cu75Ni25 alloy upon contact assessment of $\begin{aligned} & \text { with the skin is about twice as } \\ & \text { high as that of pure nickel. If for }\end{aligned}$ both the copper-nickel and purerelative allergy risks nickel coins all of the transferred contamination consists of slowly requires further dissolving compounds or particles, this factor of two will investigation compensate the twofold reduction in the amount of nickel transferred to the fingers (table 1). The allergic risk of the 1 - and $2 €$ coins should then be comparable to that of pure-nickel pieces of similar size. If all contamination from pure nickel coins dissolves slowly, while that from copper-nickel alloys contains a rapidly dissolving component, the 1 - and $2 €$ pieces may represent a higher risk than pure-nickel coins. This rather surprising conclusion would of course equally apply to any other copper-nickel coin.

A more precise assessment of relative allergy risks requires further investigation into the nature and solubility rates of metallic contamination resulting from the handling of coins. Preliminary results obtained by energy-dispersive electron-beam microscopy confirm the contribution of particulate matter. This is illustrated by fig.4, which shows micrographs of conductive tape that has been in contact with the border area across the two alloys of a $2 €$
Total amounts collected in patch tests (skin + coin)

- Ni a Cu C $\mathrm{Zn}$

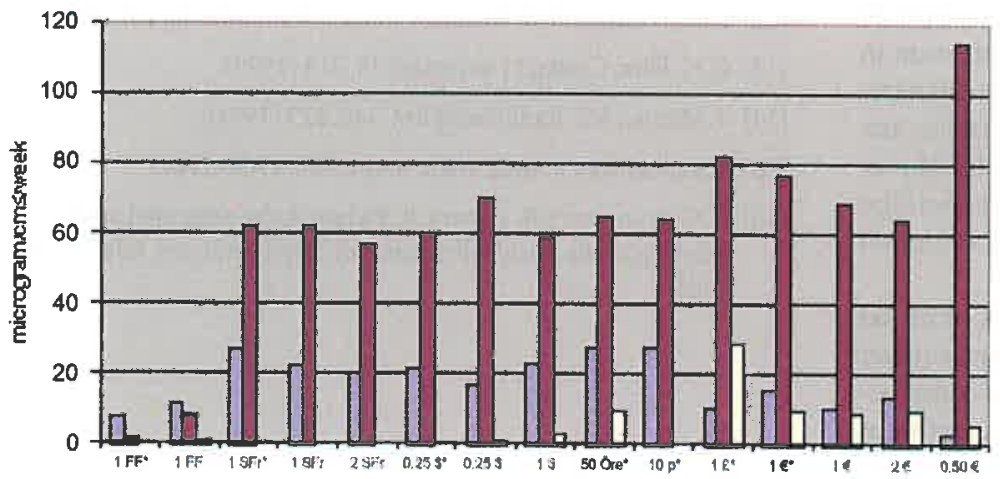

1 Fig. 3: Metal release rates determined by taping polished coins to the skin for a duration of $24 \mathrm{~h}$ (data labelled with an asterisk*) or $72 \mathrm{~h}$ They were obtained by adding the metals sampled from the imprints left on the skin and those from the face of the coin that had been in contact with the skin. Their respective contributions were in a ratio of about 3 to 1 . The release rates are expressed in $\mu \mathrm{g}$ per $\mathrm{cm}^{2}$ and per week, where the surface considered is the geometric area of one face of the coin. 

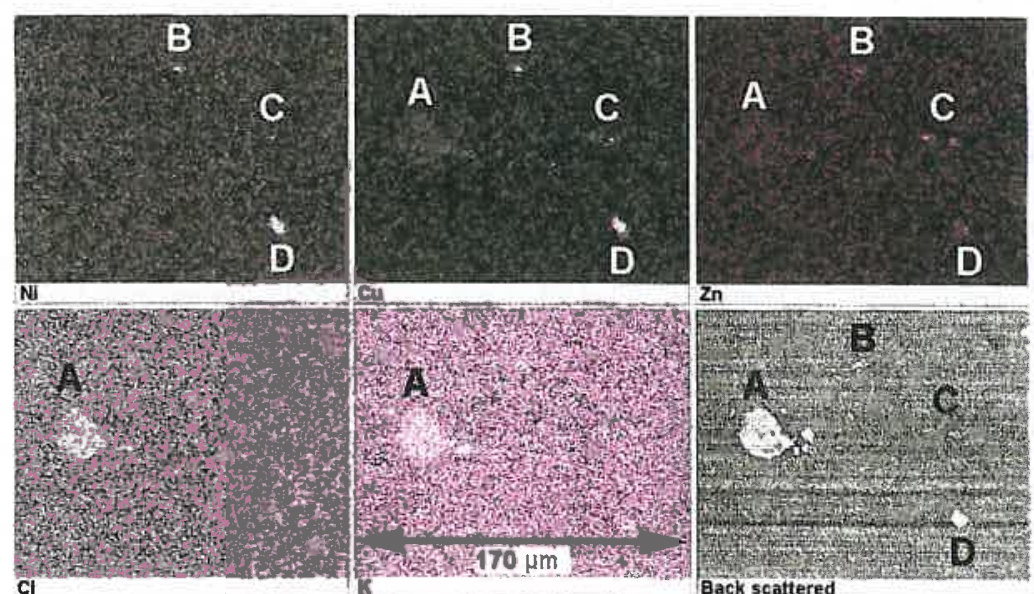

D

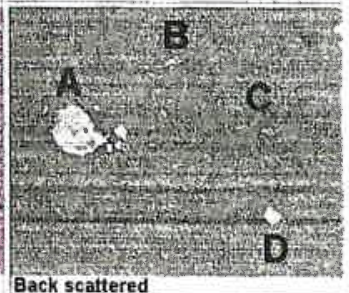

Fig. 4: Backscattered electron scan and secondary electron micrographs of adhesive tape that has been in contact with the border area encompassing the two different alloys of a $2 €$ coin (Cu75Ni25 and Cu75Zn20Ni5). The compositions of the labelled features are: $\mathrm{A}=\mathrm{Na} 37 \%, \mathrm{~K} 25 \%, \mathrm{Ca} 3.5 \%, \mathrm{Cl} 32 \%$; B and $\mathrm{D}=\mathrm{Cu} 76 \%$, Ni $23.5 \%$; C $=75.5 \%$, Zn $19.5 \%$, Ni $4.3 \%$. Feature $A$ is probably a skin and/or sweat residue, while $B, C$ and $D$ are metallic particles with the same composition as the coin's alloys. piece. Several $\mu \mathrm{m}$-size particles are seen $B, C$ and $D$, whose composition closely matches that of the coin's Cu75Ni25 and Cu75Zn20Ni5 alloys. The largest object, labelled "A", is probably a sweat and/or skin residue [16].

\section{Conclusions}

The two nickel-containing 1 - and $2 €$ coins do contribute to the contamination of the fingers by nickel and this contamination can be reliably evaluated by manipulation tests. The amounts of metallic species that pre-exist on the surface of coins and which represent a reservoir accessible to contamination by handling, can be quantified by polishing the pieces and analysing the metals thus sampled. The amount of nickel accessible on pure-nickel or Cu75Ni25 copper-nickel surfaces is of the order of $1 \mu \mathrm{g} / \mathrm{cm}^{2}$, while it represents about $0.5 \mu \mathrm{g} / \mathrm{cm}^{2}$ averaged over the surface of the bi-metallic euro coins. Typically, a single manipulation of a used nickel or copper-nickel coin with a face area of about $10 \mathrm{~cm}^{2}$ releases $\approx 0.5 \mu \mathrm{g}$ of nickel, while $\approx 0.3 \mu \mathrm{g}$ is transferred to the fingers when handling a $1-$ or $2 €$ piece. Taking into account published exposure thresholds [14] these quantities imply that skin concentrations sufficient to provoke allergic reactions will be reached upon manipulation of several hundred coins. Compulsive manipulation of a same coin is unlikely to reach the non-occluded threshold of $15 \mu \mathrm{g} / \mathrm{cm}^{2}$ [14], since the contribution of each piece is limited by the accessible "reservoir", which is of the order of $1 \mu \mathrm{g}$ per coin, distributed over $10 \mathrm{~cm}^{2}$ finger area.

When taped to the skin, even previously polished $1-$ and $2 €$ coins will provoke, after several hours, a nickel concentration in excess of $1 \mu \mathrm{g} / \mathrm{cm}^{2}$. In prolonged patch tests, these coins will therefore produce allergic reactions with sensitized people, just like any other nickel-or copper-nickel piece, as clearly shown by Aberer and Kränke [3]. While such tests confirm that the bi-metallic euros behave similarly to other copper-nickel pieces, they do not represent evidence for an increased allergic risk.

It is therefore quite legitimate to claim that the risk of nickel allergy due to the manipulation of coins could have been further reduced by not incorporating any nickel in the surface composition of the common currency. There is, however, no pertinent evidence to support the much publicised notion that the introduction of the euro has increased the risk of nickel allergy.

\section{Acknowledgments}

We are indebted to the Comité Mixte Inter-Universitaire FrancoMarocain (Grant AI 99/186F/SM and Grant MA 02/39), to Scientartphie Environnement, and to the Marionnaud company for financial support.

\section{References}

[1] A.L.Friedberg, The Numismatist, April 2002, 384.

[2] C. Lidén, S. Carter, Contact Dermatitis 44, 160 (2001).

[3] W. Aberer, B. Kränke, British J. Dermatology 146, 155 (2002).

[4] F.O. Nestle, H. Speidel, M.O. Speidel, Nature 419, 132(2002).

[5] C. Westney, Nature Science Update, Sept. 12,(2002)

[6] The Economist, Sept. 14, 2002.

[7] P.-G. Fournier, T.R. Govers, J. Fournier, M. Abadi, C.R. Physique 3, 749 (2002).

[8] European Parliament and Council Directive 94/27/EEC, JO CE L 188,1 (1994).

[9] European Committee for Standardisation (CEN), JO CE C 205, 5 (1999): EN 1810 (1998), EN 1811 (1998), EN 12472 (1998).

[10] N.B. Pedersen, S. Fregert, P. Brodelius, B. Gruvberger, Acta Dermatovener 54, 231 (1974).

[11] P.-G. Fournier, T.R. Govers, M. Abani, H. Boughaleb, M. Monkade, Phys. Chem. News 6, 82 (2002).

[12] P.-G. Fournier, T.R. Govers, Contact Dermatitis 48, 181 (2003).

[13] G.N. Flint, Contact Dermatitis 39,213 (1998).

[14] T. Menne, Sci. Total Environm. 148, 275 (1994).

[15] J. Stephenson, J. Amer. Med. Assoc. 288, 1708 (2002).

[16] P.-G. Fournier, T.R. Govers, R. Pichon, to be presented at the General Meeting of the Société Française de Physique, Lyon, July 7 - 10, 2003. 